DOI: https://doi.org/10.54103/1972-9901/16694

\title{
DOMENICA ROMAGNO
}

\section{Fra rappresentazioni concettuali, categorie grammaticali e accidenti della storia: «il fascino inquieto della ricerca scientifica» di Romano Lazzeroni}

\author{
«Non vive ei forse anche sotterra, quando \\ gli sarà muta l'armonia del giorno, \\ se può destarla con soavi cure \\ nella mente de' suoi?» \\ (Ugo Foscolo, Dei sepolcri)
}

\begin{abstract}
Between conceptual representations, grammatical categories and historical accidents: the "restless fascination of scientific research» of Romano Lazzeroni. This paper discusses the impact of Romano Lazzeroni's scholarship on Historical Linguistics and Indo-European studies altogether, by analyzing his ground-breaking works and long-lasting legacy in relation to the state of the art and current research on four main questions: 1. morphosyntax/semantics interface; 2. linguistic and cultural reconstruction; 3. linguistic categorization; 4. language change. A portrait of Romano Lazzeroni as a brilliant and unique Teacher is also provided, as a modest token of gratitude to a giant of science and big-hearted Mentor.
\end{abstract}

KEYWORDS: Historical Linguistics, Morphosyntax/Semantics Interface, Linguistic Categorization, Indo-European Reconstruction, Language Change.

1. La ricerca scientifica di Romano Lazzeroni: principi e prospettive*

La produzione scientifica di Romano Lazzeroni è vastissima: comprende temi, metodi e lingue diversi, toccando quasi tutti i campi del sapere linguistico. Scelgo

\footnotetext{
* Questo lavoro è stato presentato in forma di relazione, il 27 novembre 2020, durante la seduta del Sodalizio Glottologico Milanese in ricordo di Romano Lazzeroni. Ringrazio Patrizia Bologna e tutti i cari amici del Sodalizio Glottologico Milanese, per avermi invitata a parlare di Romano Lazzeroni scienziato e maestro, in una sede che tante volte lo ha visto protagonista e che gli era particolarmente cara. Ringrazio
} 
quattro linee di ricerca, che testimoniano del modo in cui Romano guardava alla lingua e, cioè, come a un sistema governato da algoritmi, dipendente tanto da variabili sociostoriche quanto da principi neurocognitivi: l'interfaccia fra semantica e morfosintassi, la ricostruzione linguistica come ricostruzione culturale, il rapporto fra categorie e categorizzazione, il mutamento linguistico.

«Dire che l'oggetto della linguistica deve essere la lingua e questa soltanto - ha scritto Romano nel suo ricordo di Walter Belardi (Lazzeroni 2011: 58) - è come creare una barriera intorno a una disciplina che non ammette barriere». Romano Lazzeroni ha praticato l'interdisciplinarietà, la multidisciplinarietà, la compenetrazione dei saperi assai prima che queste espressioni diventassero etichette indispensabili per qualsiasi call for papers o progetto di ricerca che avesse una qualche possibilità di finanziamento. E questo certo - ce lo ha insegnato Romano - dipende direttamente dall'oggetto della nostra ricerca, il linguaggio della parola, all'intersezione fra natura e cultura (Lazzeroni 2005: 22). Ma è anche indissolubilmente legato alla sua unica e inimitabile ironia, da intendersi come capacità di cambiare punto di osservazione (e, quindi, di aprire strade nuove), di mantenere in equilibrio piani prospettici diversi (e, quindi, di trovare un ordine nel caos apparente), di ripensare realtà date per acquisite: «la verità non si accetta - sono parole di Romano - il conformismo è il silenzio della ragione, ma si cerca, e si cerca sempre e tenacemente, anche quando si ha paura di trovarla».

2. L'interfaccia tra semantica e morfosintassi: sistema verbale e codifica argomentale nelle lingue indoeuropee antiche

Il complesso sistema di relazioni fra dimensioni funzionali e codifica morfologica e sintattica è costantemente al centro degli studi di Romano Lazzeroni, per oltre sessant'anni. In uno dei suoi primi lavori, del 1956, undici anni prima del fondamentale articolo di Vendler (Vendler 1967) sulle classi verbali e venti anni prima del noto volume Aspect di Comrie (Comrie 1976), egli si interrogava sul rapporto fra aspetto verbale e frase negativa in greco antico (Lazzeroni 1956), cogliendo acutamente la distinzione - che gli strumenti teorici di allora rendevano assai più sottile di adesso -

Luca Alfieri, Emanuele Banfi, Marina Bartolotta, Lorenzo Benedetti, Marina Benedetti, Laura Biondi, Patrizia Bologna, Maria Piera Candotti, Eleonora Cattafi, Michela Cennamo, Francesca Chiusaroli, Paola Cotticelli, Francesca Cotugno, Federica Da Milano, Alessandro De Angelis, Valeria De Rose, Francesco Dedè, Francesca Dovetto, Claudia Fabrizio, Franco Fanciullo, Anna Giacalone Ramat, Giulia Greco, Brian Joseph, Leonid Kulikov, Umberto Laffi, Kristen Leone, Giuseppe Longobardi, Marco Mancini, Carolina Marescotti, Giovanna Marotta, Lucio Melazzo, Andrea Moro, Edoardo Nardi, Adriana Perrelli, Cecilia Poletto, Diego Poli, Anna Pompei, Flavia Pompeo, Paolo Ramat, Giovanna Rocca, Velizar Sadovski, Emanuela Sanfelici, Andrea Scala, per i commenti al testo. La responsabilità di quanto è scritto è interamente mia. 
fra punto di vista sull'evento e proprietà semantiche inerenti al lessema. Attraverso lo spoglio completo delle opere di Erodoto, Sofocle, Aristofane, Platone, Eschine, Demostene, Isocrate e Tucidide, avvalendosi di metodi statistici per l'analisi qualitativa e quantitativa dei dati, Romano Lazzeroni giunge alla conclusione che in greco antico, come in altre lingue, la negazione neutralizza la distinzione fra evento rappresentato come giunto a compimento, nella sua totalità, ed evento visto nel corso del suo svolgimento, ma che «l'originalità del greco rispetto alle altre lingue» - la sua attenzione, qui, è rivolta principalmente alle lingue slave - «consiste nel fatto che questa condizione è rappresentata non solo sul piano lessicale, ma anche su quello morfologico in relazione all'impiego di categorie grammaticali per esprimere nozioni di aspetto» (Lazzeroni 1956: 229).

La correlazione fra negazione e telos dell'evento è stata studiata, in anni più recenti, con prospettive e metodi diversi (cfr. Hopper - Thompson 1980, fra gli altri), nell'organizzazione, nel mutamento e nell'apprendimento delle lingue, da quelle slave (Slabakova 2001: 93 ss.; Lindstedt 1995; Willis 2013) a quelle romanze (Manzini Savoia 2011: 80 ss., 128 ss.), dal greco antico (Romagno 2002, 2005: 27 ss.), al cinese (Song 2018), ai dialetti italiani meridionali (Romagno 2015).

In quello studio del 1956 - preceduto solo da una nota di Vendryes (secondo cui «il semble qu'il y aurait contradiction à nier ce qui est en même temps affirmé comme accompli»: Vendryes 1946: 11) e da qualche sporadica osservazione di Brunel, contenuta nella monografia sulla preverbazione in greco attico $(1939: 81,98,139)$, in cui le nozioni di concreto, perfettivo e telico tendono a confondersi - Romano Lazzeroni, per primo, pone l'attenzione sulla necessità di analizzare l'alternanza fra evento affermato ed evento negato in relazione non solo alle distinzioni di semantica verbale, ma anche alle categorie morfologiche che di quelle distinzioni rappresentano strategie di codifica complementari. Nella ricerca di Romano Lazzeroni, fin dal 1956, la volontà di combinare prospettive e metodi diversi non è mai disgiunta dall'attenzione alla lingua come sistema: segno della sua originale grandezza, questo tratto è frutto di solidissimo sapere, libertà di pensiero e rigore nell'azione.

Lo studio su aspetto e negazione in greco antico - insieme a quello, ad esso complementare, del 1957, sulla combinabilità di forme verbali diverse con gli avverbi di rapidità e con quelli significanti "improvvisamente" (Lazzeroni 1957) - apre una strada nuova nell'indoeuropeistica, percorsa da un numero via via crescente di studiosi, in Italia e fuori dall'Italia, e riconosciuta ancora oggi come indispensabile per svolgere uno dei compiti fondamentali della linguistica storica, e indoeuropea in particolare: affrontare i problemi ereditati dalla tradizione alla luce delle acquisizioni teoriche e sperimentali contemporanee.

Lungo questa strada, e con una particolare attenzione rivolta alle categorie del verbo e alla codifica degli argomenti nucleari del predicato, Romano Lazzeroni ha ricostruito un sistema di corrispondenze fra le lingue indoeuropee più anticamente attestate, in cui ogni elemento è motivato per se e, insieme, indispensabile a dar ragione degli altri. Questo sistema si basa principalmente - ma non esclusivamente - sui dati del greco e dell'indo-iranico (vedico e avestico, in particolare): ciò si motiva in un principio di omogeneità dei sistemi verbali e di codifica argomentale fra queste lingue indoeuropee 
antiche (sul cui ruolo nella ricostruzione indoeuropea basterà citare, insieme a quelli di Romano, gli studi di Jakob Wackernagel, Johanna Narten e Helena Kurzová: cfr. Wackernagel 1904, 1926; Narten 1964, 1968; Kurzová 1999, fra gli altri), che non si applica, in ugual misura, ad altre lingue.

Attraverso una serie di studi ${ }^{1}$, inaugurata dal capolavoro del 1977 - che ha rivoluzionato la ricerca sul sistema verbale indoeuropeo e che mostra, fin dal titolo (Fra glottogonia e storia: ingiuntivo, aumento e lingua poetica indoeuropea), il tratto caratteristico di Romano: asciutto, incisivo, non convenzionale - partendo dall'analisi dei risultati di un celebre lavoro di Thurneysen (1885), in parte ripresi anche da Kuryłowicz $(1932,1964)$ e Stang (1932), egli ha mostrato che in una fase arcaica del sistema verbale greco e indo-iranico (o, meglio: nella tradizione indoeuropea confluita nel sistema verbale del greco e dell'indo-iranico), il rapporto fra lessema verbale e tempo non è espresso grammaticalmente. Il sistema si basa sull'opposizione fra rappresentazione del processo e rappresentazione dello stato. La codifica grammaticale del tempo si attua mediante la progressiva grammaticalizzazione di due particelle deittiche: di $-i$, segno della deissi prossimale, (per cui, ad esempio, in sscr. la forma marcata bharati si oppone alla più antica bharat) e, in una parte del mondo indoeuropeo che comprende il greco, l'indo-iranico e l'armeno, di $*_{e-}(>$ indo-iranico $a$-, il cosiddetto "aumento"), probabile segno della deissi distale (per cui $\underline{a}$-bharat affianca - e gradualmente sostituisce - bharat, in opposizione a bharati ).

La codifica morfologica del tempo grammaticale innesca una complessa serie di mutamenti che offusca la situazione originaria. Tracce di quella situazione sono, tuttavia, visibili in fase storica e non sono sfuggite al genio di Romano Lazzeroni: come egli ci ha insegnato «il mutamento linguistico comporta un processo graduale in cui il vecchio convive, spesso a lungo, col nuovo» (Lazzeroni 2015a: 8).

Il modello di sistema verbale indoeuropeo così ricostruito consente di risolvere, in un quadro unitario e coerente, un'ampia serie di "enigmi" dell'indoeuropeistica; a cominciare da quello che unisce i tre elementi, apparentemente irrelati, che compongono il titolo del capolavoro del 1977 (cfr. anche Lazzeroni 1980, 1982, 1984, 2017b). "Ingiuntivo" è termine coniato da Brugmann in riferimento a quelle forme, con desinenze primitive (impropriamente dette "secondarie") e senza aumento, attestate più stabilmente in vedico e in avestico che in Omero (Hoffmann 1967). Sul piano del contenuto, l'ingiuntivo mostra una pluralità di valori, non modali e modali, a lungo apparsi inconciliabili: esso infatti, ha le desinenze dei tempi storici, ma ha anche valore di presente; non ha l'aumento, ma ha anche valore di passato; non ha marche modali, ma ha anche valore modale. Ponendo rigorosamente la prospettiva diacronica al centro 
della sua analisi, Romano Lazzeroni ricostruisce la sequenza degli eventi che dà ragione, formalmente e funzionalmente, dei dati della comparazione:

- la rappresentazione del processo metacronico era codificata dall'ingiuntivo attivo;

- una volta formatosi il tipo *bhereti (sscr. bharati), con il valore di presente attuale, l'ingiuntivo *bheret (sscr. bharat) diventa segno della non attualità e, quindi, del presente metacronico (conservando, in questo, il valore dell'antico indicativo) e del passato e, in opposizione al nuovo indicativo con $-i$, assume valori modali. Il vedico e l'avestico (in cui l'aumento è quasi assente) conservano tracce di questa fase;

- l'ingiuntivo, segno del passato, viene, poi, caratterizzato con l'aumento: le forme aumentate si distinguono dalle corrispondenti non aumentate, in quanto codifica preteritale, in opposizione a valori metacronici e modali. Questa fase corrisponde, sostanzialmente, alla situazione del RigVeda;

- l'ingiuntivo privo di aumento, come segno del presente metacronico, scompare sotto la pressione delle nuove forme di presente con $-i$. L'opposizione comprende due unità funzionali, di cui una conosce due varianti formali: e.g., bharati (presente) vs. $\underline{a}$-bharat/bharat (passato). Questa fase corrisponde alle situazioni omerica e - con qualche differenza - atharvavedica;

- il tipo bharat (senza aumento) scompare e l'opposizione rimane fra bharati (presente) e $\underline{a}$-bharat (passato). Questa fase corrisponde ai dati del sanscrito classico, dell'antico persiano e del greco classico.

Diventa chiaro, allora, che l'uso facoltativo dell'aumento in Omero non è da attribuirsi alla lingua poetica indoeuropea (su cui si veda Wackernagel 1926: 212 ss.), ma «consegue alla sopravvivenza dell'ingiuntivo nella tradizione conservatrice della poesia» (Lazzeroni 1977: 30). Le tavolette micenee, che di poetico hanno ben poco, confermano che in greco l'aumento può mancare anche in prosa.

Molti altri tasselli definiscono la solidità di questo sistema, in cui "tutto si tiene" interlinguisticamente, rivelando ampi pezzi, e fondamentali, della tradizione indoeuropea confluita nelle lingue storiche. Gli studi di Romano Lazzeroni:

- hanno individuato la motivazione, diacronicamente fondata sul piano formale e funzionale, della distribuzione delle diverse classi di presente e del loro rapporto con il tema dell'aoristo: nelle lingue che - come il greco e l'indo-iranico conoscono l'opposizione fra tema del presente/imperfetto e tema dell'aoristo, «il polimorfismo dei rispettivi temi è conseguente alla significazione morfologica del tempo grammaticale» (Lazzeroni 2011c: 49). Mi limito, qui, a citare le illuminanti pagine sulla classe radicale (Lazzeroni 1980, 1983a, 1985b, 1993, 2008), sul ruolo di *-ye-/-yo- (indo-iranico -ya-) nella significazione dell'anticausativo (Lazzeroni 2002a, 2004, 2017a), sulla codifica di transitivi e causativi (Lazzeroni 2009, 2017c, cfr. anche 2011b), sulla soluzione all'“enigma" di Kuiper (Lazzeroni 2008);

- hanno rovesciato la prospettiva della arcaicità del sistema verbale dell'ittita, 
chiarendo la filiera che ha prodotto il paradigma monotematico (in cui presente e preterito sono formati dallo stesso tema: e.g., etmi "mangio $>* e d-m i$, preterito etun): la non distinzione fra imperfetto e aoristo in ittita «non discende da una fase preindoeuropea o indoittita, bensì consegue alla relativamente recente morfologizzazione del tempo grammaticale» (Lazzeroni 2011c: 51). In sostanza, su ogni ingiuntivo radicale si è formato un presente e sono mancati gli ingiuntivi residui destinati a fornire l'aoristo. In questo quadro, si svela anche il mistero (cfr. Di Giovine 1996, Jasanoff 2003 e Rose 2006, con bibliografia ivi citata) della coniugazione in - $h i$ dell'ittita, la cui formazione Romano Lazzeroni pone in rapporto con il perfetto "risultativo", quello - cioè - seriore, che significa lo stato dell'oggetto (Lazzeroni 2011c);

- hanno definito le basi per la comprensione dello sviluppo del medio indoeuropeo che, da categoria derivazionale (nel senso di Bybee 1985), diventa categoria flessiva, convogliando nuovi valori: «in un sistema fondato sull'opposizione dei tempi - ha scritto Romano Lazzeroni (1990: 22) - erano contenute le premesse perché le due diatesi, unite dal tratto della temporalità, diventassero, tendenzialmente, simmetriche»;

- hanno fornito contributi fondamentali alla comprensione del rapporto fra medio e perfetto (Lazzeroni 1990, 2014; cfr. Neu 1968, con particolare riferimento all'ittita). Alla rappresentazione del processo metacronico, veicolata dall'ingiuntivo, si opponeva la rappresentazione dello stato, veicolata dal perfetto (Lazzeroni 1977, 1990, 2014). Perfetto e medio, connessi sul piano formale (si pensi alla somiglianza delle due serie arcaiche di desinenze: Kuryłowicz 1932; Stang 1932; Watkins 1969; Di Giovine 1996: 221 ss.), veicolano la nozione di stato del soggetto, ma la loro originaria distribuzione complementare, conservata in vedico (Di Giovine 1990-1996) e in greco (Romagno 2005), si motiva nell'espressione grammaticale di contenuti noetici diversi (Lazzeroni 1990): dinamico il medio, prototipicamente inaccusativo; non dinamico il perfetto, prototipicamente stativo qualificativo (Romagno 2002, 2005, 2014, 2021), e rimasto, infatti, a lungo estraneo al processo di grammaticalizzazione del tempo;

- hanno offerto la soluzione definitiva della dibattuta questione della terza diatesi (cfr. Oettinger 1976, Jasanoff 1978), riconosciuta, oltre che nell'indo-iranico (Kümmel 1996), nel cosiddetto "medio di tipo - $a$ " dell' ittita (Watkins 1969: 84): Romano Lazzeroni ha dimostrato che le desinenze cosiddette "stative" non appartengono a una terza diatesi distinta dal medio, ma sono le desinenze originarie del medio, precedenti alla sua attrazione nel sistema dei tempi e alla conseguente ristrutturazione delle sue desinenze sul modello di quelle attive del presente: esse, infatti, corrispondono al prototipo funzionale della categoria, che ha resistito più a lungo alla ristrutturazione (Lazzeroni 1990, 1993b, 2014, 2015a).

Questi sono soltanto alcuni degli “enigmi” risolti dalla «semplicità dell'intelligenza sistemica» di Romano Lazzeroni - per riprendere la bellissima definizione di Marco Mancini (2020: 23). Di molti altri potremmo parlare. Aggiungo soltanto che all'interno 
del modello lazzeroniano di sistema verbale indoeuropeo hanno operato e continuano a operare numerosi studiosi, in Italia e fuori dall'Italia, giungendo, in modo indipendente, a risultati robustamente coerenti in lingue diverse: per citare solo alcuni dei tanti lavori che possono ricondursi al modello lazzeroniano, ricordiamo le ricerche di Stephanie Jamison (1983) e di Leonid Kulikov (2012a, 2012b) sul sanscrito e quelle di Helena Kurzová $(1993,1999,2011,2014)$ su greco, latino e altre lingue indoeuropee antiche.

\subsection{Fra ruoli semantici, funzioni sintattiche e proprietà del referente}

La comprensione dei principi che soggiacciono all'organizzazione e al mutamento del sistema verbale nelle lingue indoeuropee antiche apre una strada nuova nello studio delle strategie di codifica argomentale di queste lingue: in particolare, dell'alternanza fra codifica "canonica" e codifica "non canonica" (cfr. Aikhenvald et al. 2001). Il solido modello lazzeroniano, che non ammette sbavature e deviazioni, e in cui il rispetto per i dati non è mai disgiunto dalla riflessione teorica e dalle osservazioni tipologiche, consente di far luce su una serie di fenomeni, apparentemente distanti, che si riconducono a un principio "universale" (tipologico e cognitivo: cfr., fra gli altri, Dixon 1994): le lingue del mondo non manifestano "purezza" tipologica; la motivazione dei fenomeni che si discostano dal tipo dominante andrà cercata nella tensione fra rappresentazione semantico-concettuale e pressione sistemica (cfr. Cennamo 2001; Romagno 2011; fra gli altri). Gli studi di Romano Lazzeroni (Lazzeroni 1995a, 2002b, 2002c, 2013a, 2015b, 2017d) dimostrano che anche nelle lingue indoeuropee antiche la codifica degli attanti - questione a lungo dibattuta (cfr. Uhlenbeck 1901; Villar 1984; Rumsey 1987; Cuzzolin 1998; Drinka 1999; Dardano 2013; Pooth et al. 2019) - è regolata da meccanismi di interazione (e talora di conflitto) fra ruoli semantici, ruoli sintattici e proprietà del referente (in particolare, animatezza e individuazione). Mi limito a citare i geniali contributi sui metaplasmi di genere, sull'origine del neutro tematico (o debole), sulla codifica non canonica del soggetto (cfr., in particolare, Lazzeroni 2002b, 2002c). Attraverso un'ampia ricerca, che spazia dal sanscrito al greco, dall'iranico all'ittita, dal latino alle varietà romanze, Romano Lazzeroni ha mostrato che:

- la selezione del genere (animato vs. inanimato) è in relazione con il ruolo semantico dell'argomento corrispondente, rispettivamente, al soggetto o all'oggetto;

- indipendentemente dalla selezione del genere, il soggetto all'accusativo (caso tipico dell'oggetto, inanimato e inattivo, ma non del soggetto, animato e attivo) si configura come strategia di codifica su base semantica: in sostanza, il soggetto non canonico, in quanto semanticamente inattivo, prende il caso canonico dell'oggetto;

- i neutri deboli - e solo questi - conoscono frequentemente varianti di genere animato della stessa classe flessiva; le varianti di genere animato dei neutri atematici o forti (i più antichi), invece, si accompagnano sempre a metaplasmo

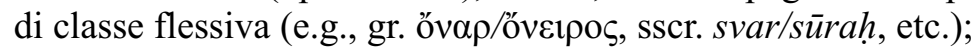

- il paradigma dei neutri deboli - diversamente da quello dei neutri forti - diffe- 
risce dal paradigma dei nomi deboli di genere animato per il solo nominativo (in sanscrito il vocativo dei neutri deboli è di genere animato);

- i neutri deboli, in conclusione, corrispondono a una classe di antichi nomi tematici di genere animato diventati neutri in seguito alla generalizzazione dell'accusativo come caso del soggetto: «il nominativo neutro tematico - ha scritto Romano Lazzeroni (2002b: 317) - è omofono dell'accusativo tematico animato perché è l'accusativo tematico animato».

\section{Ricostruzione linguistica e ricostruzione culturale}

Gli studi di indoeuropeistica su sistema verbale e codifica argomentale hanno beneficiato dell'amore di Romano per il vedico; egli è certamente uno dei maggiori rappresentanti della linguistica indiana, e vedica in particolare: come sottolineato nella motivazione del premio internazionale per l'orientalistica, di cui fu insignito nel 1999 - quello noto come "Premio Gonda", dal nome del celebre studioso a cui fu conferito nel 1988 - i suoi contributi rivelano una capacità straordinaria di interpretare i testi vedici - come è noto, difficilissimi - e di ricondurli alla cultura di cui sono espressione, combinando rigore filologico, riflessioni teoriche e osservazioni tipologiche. Di questo sono testimonianza, insieme a tanti altri studi di Romano Lazzeroni, i saggi raccolti nel volume La cultura indoeuropea (del 1998, tradotto anche in portoghese: Lazzeroni 1999b). Mi limito qui a citare due di quei capolavori: quello sull'organizzazione degli esseri viventi e inanimati, e quello sul nettare e l'ambrosia e la nozione indoeuropea della morte. Nel primo, si ricostruisce un sistema concettuale, in cui il movimento definisce la vita e il piede è simbolo del movimento. La corrispondenza della tassonomia vedica con quella iguvina rappresentata nella a lungo dibattuta formula "bipedi e quadrupedi" si comprende soltanto in un campo semantico fondato sulla rappresentazione del mondo animato come "ciò che si muove, che va" (jágat-, termine prevedico) e del mondo inanimato come "ciò che sta fermo" (sthā-). Sia in vedico sia in sanscrito classico, il "bipede" è l'uomo e mai l'uccello: non i piedi, infatti, ma le ali sono l'organo di locomozione di quest'ultimo. L'esclusione degli uccelli dal novero dei "bipedi" è, invece, inimmaginabile nelle culture greca e romana. Eppure, la comparazione dimostra che la formula è indoeuropea. La lucida analisi dei testi vedici condotta da Romano Lazzeroni ci restituisce un complesso sistema cosmogonico e di organizzazione degli esseri viventi e inanimati in cui questa formula chiaramente si motiva. E allora, possiamo concludere che è indoeuropeo tutto il sistema concettuale di cui questa formula è parte. Relitti di questo sistema, che nella tradizione vedica è organico e coerente, sopravvivono in aree diverse, adattati (in iranico), reinterpretati (in greco), fossilizzati (in umbro). La formula "bipedi e quadrupedi", che compare anche, al dativo plurale, nelle Tavole Iguvine, come parte di una preghiera (dupursus peturpursus), non è, dunque, da interpretarsi come relitto di "lingua poetica indoeuropea", ma come espressione motivata di una visione del mondo accolta dalla poesia. 
Il saggio su immortalità e morte svela il mistero del doppio antidoto per la vita

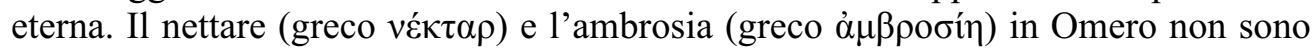
distinti per funzione o modo di assunzione e ricorrono in coppia. Ancora una volta, il dialogo tra linguistica e filologia e, quindi, l'interpretazione della memoria storica tramandata nei testi consente a Romano Lazzeroni di ricostruire un sistema di corrispondenze fra lingue indoeuropee diverse (in particolare, sanscrito, greco e latino),

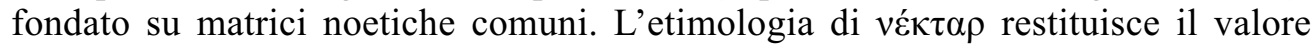
descrittivo di ciò che "attraversa" (e perciò vince) "la morte" (v\&k-, cfr. latino nex,

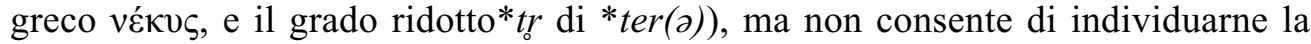
motivazione. A questa si giunge riconsiderando i dati greci alla luce dell'analisi dei testi vedici e della tradizione latina. Il pensiero vedico distingue la morte naturale da quella prematura: la prima è prodotta da vecchiaia, la seconda da qualsiasi altra causa (nell'AV sono 100 le morti innaturali: cf. II, 28, 1). Solo la morte prematura è un male da scongiurare e da vincere, e vincerla è rappresentato come un "attraversarla" (tarati). All'altro margine del mondo indoeuropeo, in latino (arcaico e classico), nex è termine marcato, che indica la morte prematura, in opposizione a mors, che denota la morte

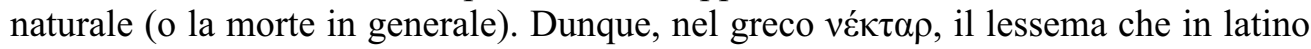
designa la morte prematura si combina con la base verbale *ter(a) che in vedico si riferisce alla vittoria su questa morte. La rappresentazione concettuale è, evidentemente, indoeuropea. Perché, poi, tale vittoria venga descritta come un "attraversamento" ce lo dice il vedico. Nella cultura vedica il male - e quindi la morte, il sommo dei mali - è rappresentato come una strettoia (amhas-). La vittoria sul male è un "attraversamento" della strettoia. E ancora: il nome greco dell'ambrosia contiene il nome della morte naturale: $\alpha \mu \beta \rho o \sigma i ́ n<* n ̊ m r t-$. Si comprende, allora, perché i due alimenti magici, il nettare e l'ambrosia, ricorrano in coppia: l'uno vince la morte prematura, l'altro la morte naturale. Nei nomi greci dei cibi degli dei si riflette, dunque, la doppia nozione indoeuropea della morte.

La tipologia belardiana del segno nell'indoeuropeo ricostruito (Belardi 1985, 1990, 1993) diventa, nell'interpretazione linguistico-filologica di Romano Lazzeroni, strumento di ricostruzione culturale.

Il riferimento al "Premio Gonda" ci consente di ricordare il senso che Romano dava a premi e riconoscimenti (che ha ricevuto in gran numero e di grandissimo prestigio): la motivazione sopracitata fu prontamente distrutta e i denari donati, senza una voce, alla biblioteca pisana di linguistica (che ospita quella che, ancora oggi, è una delle più importanti collezioni di linguistica indiana in Europa); lo spirito di Romano era incompatibile con le autocelebrazioni. Insignito dal Presidente della Repubblica Carlo Azeglio Ciampi della medaglia d'oro ai Benemeriti della scuola, della cultura e dell'arte, non partecipò alla cerimonia di consegna, né diffuse la notizia, che molti di noi appresero dai quotidiani nazionali. 


\section{Categorie e categorizzazione}

I lavori discussi fin qui rivelano una attenzione costante all'organizzazione linguistica dei dati dell'esperienza e, di conseguenza, al rapporto fra categorie noetiche e discretezza formale. Questo rapporto è al centro di molti studi di Romano Lazzeroni, che, per primo, ha introdotto la teoria dei prototipi e il modello della somiglianza familiare (Wittgenstein 1953; Berlin - Kay 1969; Rosch - Mervis 1975) nella linguistica storica indoeuropea (cfr. Lazzeroni 1990, 1995b), rivoluzionando, ancora una volta, la prospettiva su questioni antiche, rimaste a lungo irrisolte, e individuando problemi nuovi e nuove soluzioni.

Dopo secoli di riflessione (da Pānini in poi) sulla pluralità dei valori del medio indoeuropeo, la soluzione arriva nel 1990, con il cambio di prospettiva di Romano Lazzeroni, che invita a guardare alla diatesi come a una "categoria naturale" nel senso di E. Rosch (Rosch 1973, 1978; cfr. Taylor 1989), in cui è possibile individuare un centro prototipico e una periferia di costituenti legati al prototipo da un rapporto di somiglianza (Lazzeroni 1990). Ciò non solo ha risolto apparenti aporie sistemiche (a cominciare dalla nota neutralizzazione dell'opposizione di diatesi nell'imperativo: la agentività richiesta dall'imperativo era incompatibile con il prototipo del medio; la formazione dell'imperativo medio è frutto del processo che ha reso simmetriche le due diatesi), ma ha anche aperto la strada a un filone di ricerca sulla diatesi indoeuropea che continua, ancora oggi, a fornire i risultati più robusti e coerenti e a individuare gli sviluppi più promettenti.

L'applicazione del modello prototipico e di quello wittgensteiniano della somiglianza familiare all'analisi dei processi di costituzione e di espansione della categoria del genere grammaticale nelle lingue indoeuropee antiche (cfr., in particolare, Lazzeroni 1992a, 1993a) consente a Romano Lazzeroni di chiarire aspetti cruciali di quello che è stato definito «one of the still unsolved puzzles of linguistic science» (István 1959: 1) "uno degli enigmi irrisolti della scienza linguistica". In uno dei suoi lavori su questo tema, del 1993, egli ha scritto: «Molto del genere grammaticale resta enigmatico. Ma se rifletteremo sui principi costitutivi delle categorie linguistiche e sulle relazioni cognitive espresse dalle categorie grammaticali, forse il genere grammaticale ci apparirà meno fantastico della classificazione degli animali nell'Emporio celeste di Borges» (Lazzeroni 1993a: 16).

Di molti altri studi, anche in questo caso, potremmo parlare. Aggiungo soltanto una breve nota su due di essi: quello sulla vexata quaestio dei nomi indoeuropei d'agente, in *-tér e -tor (Lazzeroni 1992b) e quello sui nomi d'azione (Lazzeroni 1997, 2012a). Nel primo, l'alternanza fra le due serie, che si distinguono per la posizione dell'accento e la rezione (e in iranico anche per l'apofonia radicale) viene interpretata in relazione alla posizione che i diversi derivati occupano nelle gerarchie - interagenti - di animatezza (Silverstein 1976) e di individuazione (Timberlake 1977). Nel secondo, a cui si riconducono due lavori, del 1997 e del 2012, si mostra che il diverso comportamento morfologico e sintattico dei nomi d'azione e dei nomi d'agente si motiva all'interno di un gradiente di "nominalità" (vs. "verbalità"), ma che il grado di proto- 
tipicità di ciascuna delle due categorie si misura in relazione ai singoli parametri che definiscono il gradiente, e si manifesta in dipendenza dai vincoli strutturali imposti dal sistema (cfr., in particolare, Lazzeroni 2012a). I risultati di questo studio - come spesso $\mathrm{o}$, meglio, sempre nella ricerca di Romano Lazzeroni - hanno una portata ben più ampia di quella definita dal caso specifico e possono ricondursi a due indicazioni di metodo fondamentali: 1. le gerarchie che soggiacciono al sezionamento linguistico delle categorie noetiche sono da interpretarsi come rappresentazioni non solo multifattoriali, ma anche multidimensionali; 2 . la classificazione di una data categoria in relazione a un tratto o a una dimensione dipende dalle regole di funzionamento del sistema in cui quella categoria si manifesta morfologicamente o sintatticamente.

La definizione di prototipo include non solo la nozione di frequenza (e quella, ad essa connessa, di salienza cognitiva), ma anche la nozione di marcatezza (e quella, ad essa connessa, di estensibilità: ciò che è meno marcato è anche suscettibile di valori estensivi). La marcatezza, nelle lingue (e non solo), si manifesta come marcatezza locale: un elemento, cioè, è più o meno marcato in relazione a una data categoria o dimensione, $\mathrm{o}$ a un dato contesto morfosintattico. In ciò risiede il ruolo del cosiddetto "accordo di marcatezza", nel senso di Henning Andersen (Andersen 1980, 2001a, 2001b), cruciale nell'organizzazione e nel mutamento delle lingue. Le innovazioni, quando consistono nella creazione di una categoria, tendono a partire dagli elementi (localisticamente) meno marcati, procedendo, progressivamente verso quelli più marcati (Andersen 1990, 2001a): nella storia del sistema verbale indoeuropeo, la codifica morfologica del presente attraverso la grammaticalizzazione della particella $-i$, di cui si è parlato sopra, parte dall'indicativo, raggiunge (in modo non omogeneo fra greco e indo-iranico, e parziale) il congiuntivo e lascia indenne l'ottativo: le distinzioni di tempo sono meno prototipiche nei modi della non fattualità e della controfattualità che nell'indicativo, fattuale (Lazzeroni 1977, 2016b). Il percorso inverso, dagli elementi più marcati (meno prototipici) a quelli meno marcati (più prototipici), si osserva nella cancellazione (e fusione) di categorie: qui basterà citare la progressiva scomparsa del locativo latino (che sopravvive, però, fin nelle lingue romanze, in nomi di città, semanticamente prototipici: Lazzeroni 2005), il sincretismo di genitivo e ablativo in greco e in sanscrito (in cui coinvolge il singolare di tutte le declinazioni, salvo che - non a caso - di quella in ă, i cui temi sono di gran lunga i più frequenti: Lanman 1880), la semplificazione delle distinzioni di numero (cfr. Milizia 2013). Nella Grecia antica, lo ionico e i dialetti eolici e dorici circumegei hanno perso il duale (e non il plurale) già in epoca predocumentaria: il plurale (meno marcato e più estensivo) può includere le significazioni del duale, ma non viceversa. Se - come ha mostrato Romano Lazzeroni (Lazzeroni 1960) - la perdita del duale è un'innovazione irradiata dalle lingue dell'Anatolia antica (il greco continentale, infatti, e l'attico stesso lo conservano), diremo che la formazione di una arealità greco-anatolica, che ha dato l'avvio all'innovazione, è un accidente della storia, ma che il percorso della riduzione delle opposizioni di numero è il prodotto di un principio universale: all' "universale" tipologico di Greenberg (1963) soggiace un universale cognitivo. 
Su questi e altri fenomeni interpretabili come creazione, cancellazione o fusione di categorie, Romano Lazzeroni ha scritto pagine illuminanti (cfr., fra gli altri, Lazzeroni 2005, 2016b, 2018a, 2018b, 2019b).

L'importanza della teoria della marcatezza di Andersen nello studio del mutamento linguistico è stata ripetutamente sottolineata da Romano, che ha definito Henning Andersen uno dei più grandi linguisti dell'ultimo secolo; in modo identico Henning Andersen ha definito Romano Lazzeroni: la grande stima reciproca fra questi due giganti della linguistica è antica, anche se la loro conoscenza fuori dalle pagine scritte è avvenuta soltanto nel luglio del 2015, al ristorante Zi'Teresa di Napoli.

\section{Il mutamento linguistico}

La linea di ricerca sul mutamento linguistico è sovraordinata a quelle discusse fin qui, comprendendole tutte.

Allo stato attuale delle nostre conoscenze, gli studi di Romano Lazzeroni rappresentano il contributo più significativo alla elaborazione di una teoria olistica del mutamento, che rimane una delle principali sfide della linguistica.

A conferma del rapporto endiadico - e non certo dicotomico - fra organizzazione e trasformazione delle lingue (di cui Romano, con il suo inimitabile stile, sottolineava l'importanza fin dalla prima lezione di 'Glottologia I', e su cui Eugenio Coseriu ha scritto pagine definitive: cfr. Coseriu 1952, 1958, 1966, 1994), basterà osservare che André Martinet, uno dei padri dello strutturalismo, sceglie un punto di osservazione diacronico per la summa delle sue riflessioni teoriche (Économie des changements phonétiques "Economia dei mutamenti fonetici": Martinet 1955): il mutamento, infatti, manifesta momenti di crisi, in cui il sistema cambia e si riorganizza. La prospettiva dinamica è, probabilmente, la più adatta a svelare i principi che governano i sistemi.

Romano Lazzeroni ha posto costantemente l'attenzione sulla necessità di interpretare il mutamento linguistico in un quadro unitario e coerente, che includa tutti i livelli del sistema, e ha dimostrato che per far questo è indispensabile guardare alla lingua come a un prodotto insieme naturale e culturale (Lazzeroni 1987b, 2005): per comprendere i principi che soggiacciono all'organizzazione, al mutamento, e insieme all'apprendimento e alla perdita delle unità formali e funzionali della lingua è fondamentale distinguere ciò che è universale, in quanto neurobiologicamente determinato, da ciò che è contingente, in quanto socioculturalmente determinato.

All'interno di una rappresentazione tridimensionale del mutamento linguistico (su cui si vedano Weinreich - Labov - Herzog 1968; Belardi 1978; Trudgill 1983; Labov 2001, fra gli altri), le ricerche di Romano Lazzeroni sul mondo indo-indoiranico, greco, egeo-anatolico, italico, latino, romanzo (Lazzeroni 1967, 1968, 1969, 1972, 1984b, 1991a, 1999a, 2006, 2007, 2012b) mostrano come la variazione e il mutamento (anche quelli dovuti a interferenza: Lazzeroni 1983b; cfr. Mancini 2008, 2013) procedano non solo lungo le dimensioni del tempo e dello spazio, ma anche lungo quella della profon- 
dità socioculturale (e stratigrafica) della comunità dei parlanti; e che se è vero che il campo di elezione per lo studio del mutamento linguistico è quello delle lingue "vive", che meglio consentono di osservarlo in atto, è altrettanto vero che se si riesce ad applicare alle lingue del passato le prospettive e i metodi affinati nello studio di quelle del presente, allora - come egli ha scritto (Lazzeroni 1991b: 217) - «avremo raggiunto l'altro versante dell'insegnamento di W. Labov: capire il presente spiegando il passato».

Il modello lazzeroniano comprende l'intersezione di tre piani, a un livello di astrazione crescente e di variabilità decrescente (cfr., in particolare, Lazzeroni 2005, 2015c, 2016b, 2018a, 2018b, 2019b, 2019c, 2020):

1. il piano del rapporto tra fatti di lingua e accidenti della storia: la lingua è un sistema che si attua nella storia e perciò soggetto alle variabili della storia;

2. il piano del rapporto tra categorie linguistiche e rappresentazioni concettuali: la lingua è uno strumento cognitivo che veicola non una realtà data ma una realtà interpretata; e perciò testimonia del modo in cui i parlanti rappresentano e categorizzano il mondo, in un cronotopo di riferimento;

3. il piano del rapporto tra sistema linguistico e meccanismi neurocognitivi che ne determinano il funzionamento: la lingua è il prodotto più tipico del cervello umano (il "miracolo della parola" è ciò che definisce l'unicità dell'uomo: Maffei 2018) e perciò dipende dai principi che governano il funzionamento cerebrale.

La ricerca di Romano Lazzeroni, in cui si rispecchiano la sua visione d'insieme e la sua capacità di osservare i fenomeni cogliendone rapidamente le relazioni, individua, all'interno di questo modello, delle costanti, potremmo dire degli "universali", che definiscono i principi del mutamento (gli stessi - egli ha mostrato (Lazzeroni 2005) che soggiacciono all'apprendimento) e ne indicano i percorsi («prevedibili ma mai predicibili», perché - come ci ripeteva Romano a lezione - «tanti e tali, e impredicibili sono gli accidenti della storia»):

a. la tensione tra forme discrete e contenuti scalari: i limiti della memoria impongono che unità discrete codifichino una quantità di significati organizzati in categorie per ciò stesso scalari e dai confini sfumati e sovrapponibili. I percorsi di creazione, cancellazione e fusione di categorie, di cui si è parlato sopra, indicano direzioni possibili del mutamento (si veda anche Lazzeroni 2013b);

b. la tensione tra memoria dichiarativa, cioè la memoria di dati e di informazioni immagazzinati uno per uno, e memoria procedurale, cioè la memoria di regole o di connessioni che producono automatismi: alla base dell'organizzazione funzionale e del mutamento dei sistemi linguistici sta un principio di economia dei meccanismi della memoria. La lingua tende a organizzarsi in modo da favorire la produzione automatica delle unità linguistiche, mettendo in atto strategie che, operando a livelli più astratti delle singole unità, privilegiano 
l'elaborazione propria della memoria procedurale, a scapito dell'immagazzinamento della memoria dichiarativa (cfr. Lazzeroni 1992a, 2000b, 2015c, 2016b, 2019b, 2020).

Secondo Kiparsky (1968), il parlante tenderebbe a massimizzare il campo di applicazione di una regola, in modo da renderla produttiva nel maggior numero di casi possibile. Gli studi di Romano Lazzeroni dimostrano che la tendenza a massimizzare il campo di applicazione di una regola è, piuttosto, epifenomeno della tendenza a minimizzare lo sforzo mnemonico. Ne sono testimonianza i numerosissimi mutamenti, osservabili a tutti i livelli del sistema e in lingue diverse (già notati, ma non spiegati, in fonetica, da Paul, nei suoi Prinzipien del 1880), che procedono in modo simmetrico, passando, cioè, da un singolo costituente categoriale al taxon sovraordinato di categoria e consentendo, in tal modo, al parlante di memorizzare un'unica "nuova" regola per tutti i costituenti della categoria colpita dal mutamento (si veda, fra i molti casi, quello studiato da Giannelli 1973). In vedico, dopo il passaggio ad 'a' di 'e' e di 'o' indoeuropei (un mutamento simmetrico, certo non simultaneo, fra le due vocali: a giudicare dai prestiti arii in finnico, esso è iniziato dalla serie palatale) la declinazione dei temi in 'a' breve $(<$ i.e. $-o)$ fu riservata ai maschili, quella dei temi in 'a' lunga $(<$ i.e. $-\bar{a})$ ai femminili. Nei temi in 'a' (e solo nei temi in ' $a$ '), l'opposizione di quantità era il segno dell'opposizione di genere. Nel corso della storia del sanscrito, si è prodotta una redistribuzione del genere grammaticale sulla base della quantità vocalica, che ha investito anche gli altri timbri. Nella situazione di partenza, se un tema era in 'a' breve era maschile, se in ' $a$ ' lunga femminile; nella situazione di arrivo medio-indiana, tutti $\mathrm{i}$ temi in vocale breve furono maschili, tutti $\mathrm{i}$ temi in vocale lunga femminili (Lazzeroni 1992a).

In questo e altri fenomeni di mutamento, di cui Romano Lazzeroni ha svelato il percorso, individuandone i principi soggiacenti, lo spostamento del campo di applicazione di una regola a un livello più astratto accresce gli automatismi: il parlante è in grado di prevedere e di produrre le nuove forme senza memorizzarle una per una; basta che conosca e applichi la regola che le genera.

E ancora: molti sono i casi di ricostituzione delle "condizioni strutturali del paradigma" (nel senso di Wurzel 1984 [1989]; cfr. anche Carstairs 1987), conseguente a mutamenti fonetici o altro che ne abbiano cancellato la forma o offuscato le relazioni paradigmatiche. Quando, in latino, -os finale diventa -us (lupos > lupus) si sono create le condizioni per il collasso di II e IV declinazione: il che, a prima vista, potrebbe sembrare avvenuto. Ma Romano Lazzeroni ha mostrato che il processo è stato diverso (Lazzeroni 2000b). La IV declinazione latina sopravvive nelle lingue romanze, in un certo numero di relitti: e.g., la mano - le mano, la fico - le fico, la peco - le peco, nei dialetti italiani centromeridionali, la nuoro - le nuoro, in Campania e Calabria, e così diversi altri nomi che Rohlfs elenca nella sua Grammatica (1949-1954). Tutti questi nomi sono femminili e alcuni (ad esempio, la suoro - le suoro), anch'essi femminili, sono stati attratti nella IV declinazione dalle altre. E, perciò, nonostante il collasso dei rispettivi nominativi, le due declinazioni sono state salvate sostituendo il tratto che selezionava i rispettivi paradigmi: tutti i maschili della IV declinazione sono passati 
alla II, tutti i femminili della II sono passati alla IV, che ha conservato i propri e ha attratto alcuni nomi delle altre declinazioni. I paradigmi misti di senatus e di domus documentano il processo in atto. Come è evidente, il campo di applicazione della regola che assegnava i paradigmi non si è allargato, ma si sono sostituiti i tratti identificativi dei paradigmi, in modo da preservare il meccanismo che consente al parlante di assegnare automaticamente un lessema a una determinata classe flessionale, in base a un tratto sovraordinato a tutti i lessemi appartenenti a quella classe.

Questo principio opera non solo nella morfologia e, come si è detto, nella fonetica, ma anche nella sintassi: qui basterà citare il cosiddetto "rapporto di solidarietà", studiato, in prospettiva dinamica, da Hawkins $(1979,1983)$, ma già presente in uno degli "universali" di Greenberg (1963).

Grazie alla ricerca di Romano Lazzeroni, appare, dunque, chiaro come la deriva di Sapir (1921) e la funzionalità sistemica intesa nel senso teleologico ad essa attribuito da Croft (2000) si configurino come epifenomeni di restrizioni che hanno carattere universale, in quanto fondate su principi cognitivi e neurobiologici.

Il ruolo dei meccanismi della memoria si osserva anche nell'organizzazione delle forme cosiddette "irregolari", che devono essere immagazzinate una per una: «non sarà un caso - ha scritto Romano Lazzeroni (2019b: 178; cfr. Lazzeroni 2005: 14 ss.) - che le forme irregolari - e in particolar modo le più irregolari quali sono le forme suppletive - appartengano invariabilmente e in ogni lingua alle parole con l'indice di frequenza più alto»: la frequenza, da sola, è un potente ausilio della memoria. E infatti, quando il livello di frequenza di una data unità è basso o nullo, come nelle fasi di apprendimento e di perdita (individuale o collettiva) di una lingua, gli automatismi prevalgono, rompendo anche i confini etimologici: si producono, ad esempio, 'ando' - 'andiamo', su 'andare', come 'lodo' - 'lodiamo', su 'lodare', o 'gallo' - 'galla', sul modello di 'gatto' - 'gatta' (Antinucci - Miller 1976, Sasse 1992, Giacalone Ramat 1983, 2003: passim; Lazzeroni 2005, 2020).

Ma c'è di più: la tendenza del parlante a favorire gli automatismi, alleggerendo il carico della memoria dichiarativa, opera anche nell'organizzazione dei paradigmi irregolari o sub-regolari di parole ad alta o altissima frequenza: si pensi, ad esempio, alla partizione del paradigma di verbi italiani quali andare e uscire. Su questo fenomeno interlinguistico, con particolare riferimento alle lingue indoeuropee antiche (e moderne), Romano Lazzeroni, ancora una volta, ha scritto pagine illuminanti (Lazzeroni 2015c).

Possiamo concludere osservando che il parlante, posto di fronte a forme irregolari (o sub-regolari), siano esse prodotto di innovazione o residui, le riordina astraendo alcuni tratti comuni, uno "schema", nel senso di Bybee - Slobin (1982), che gli consenta di prevederle, e quindi di produrle, se non con certezza, almeno con un certo grado di probabilità. Certezza ( $\mathrm{e}$, perciò, automatismo nella produzione) che può crescere nel volgere della storia linguistica. Come ha mostrato Paolo Ramat (1985) il carattere scalare è proprio dell'irregolarità morfologica (e non solo): una regola, nata come probabilistica, può progressivamente diventare categorica. 


\section{La "fusione di orizzonti" nell'insegnamento di Romano Lazzeroni}

La ricerca di Romano Lazzeroni è sempre stata indissolubilmente legata alla didattica. Questo legame si basa sulla convinzione che non esiste didattica universitaria (e non solo) se non animata dallo spirito della ricerca, e sul desiderio di condividere il cammino con i più giovani. Lo abbiamo sentito dire spesso che «una delle gioie più grandi della vita è vedere gli occhi di un giovane illuminarsi per aver corso un'avventura del pensiero». In un tempo precedente a quella che Romano definiva la «burocratizzazione demenziale» dell'Università, nel Dipartimento pisano di Linguistica, animato dalla sua presenza costante, tutti, dopo un severissimo filtro iniziale, ma indipendentemente dalla posizione accademica, stavano uno accanto all'altro e lavoravano insieme, la mattina e la sera, spesso fino alle ore piccole. Grazie a Romano Lazzeroni, lo spirito di quel Dipartimento non conosce confini temporali, territoriali o nazionali, né barriere ideologiche, né contrapposizioni fra i saperi, nemmeno fra quelli cosiddetti "scientifici" e quelli cosiddetti "umanistici": come ha scritto Lamberto Maffei, grandissimo scienziato e Maestro, allievo di Giuseppe Moruzzi a Pisa e, come Romano, Accademico dei Lincei, «la distinzione tra cultura e scienza [...] è un'aberrazione priva di razionalità» (Maffei 2018: 54).

Romano Lazzeroni è sempre stato il Maestro di tutti, forse anche perché considerava tutti - e in particolare i più giovani - maestri. Riprendendo un noto avant-propos di Meillet, egli amava ripetere - lo ha ricordato recentemente Francesca Dovetto (Dovetto 2020: 80) - che gli scolari si formano da sé. Ma il suo esempio ci dimostra che gli scolari si formano grazie alla generosità dei Maestri e che la grandezza di un Maestro si misura dalla capacità di lasciare i propri scolari liberi di formarsi da sé. Romano ha sempre voluto che i propri scolari si formassero anche nel magistero di altri e che di questi si sentissero anche scolari.

Uno dei principi fondanti del suo insegnamento risiede nella consapevolezza dei limiti del nostro operare, quei limiti che - egli diceva - «alimentano il fascino inquieto della ricerca scientifica», che non ammette chiusure o immobilismi, e in cui il contributo del singolo ha ragion d'essere soltanto come anello di una catena proiettata potenzialmente all'infinito.

«La scienza non aspira, non deve aspirare, a risultati definitivi. Ogni ricerca apre la strada ad altre, che ne colmano le lacune e ne superano i traguardi. E quando un giovane raccoglie dalle nostre mani il testimone per correre a sua volta un'avventura del pensiero e, andando oltre il nostro lavoro, continua la nostra vita nella sua, allora apprezziamo compiutamente il dono che ci elargì la sorte allorché ci fece scegliere un mestiere che ci consente di vivere oltre la vita. Non omnis moriar: quello che Orazio diceva della poesia vale ancor di più per la scienza» (Lazzeroni 2019a: 13). 
Riferimenti bibliografici

Aikhenvald, A. - Dixon, R.M.W. - Onishi, M. 2001, Non-canonical marking of subjects and objects, Amsterdam, Benjamins.

Andersen, H. 1980, Morphological change: towards a typology, in J. Fisiak (ed.), Historical morphology, Berlin-New York, De Gruyter Mouton: 1-50.

— 1990, The structure of drift, in H. Andersen - E.F.K. Koerner (eds.), Historical Linguistics 1987: Papers from the 8th International Conference on Historical Linguistics, Lille, August 30-September 4, 1987, (Current Issues in Linguistic Theory 66), Amsterdam, Benjamins: 1-20.

2001a, Actualization. Linguistic change in progress, (Current Issues in Linguistic Theory, 219), Amsterdam-Philadelphia, Benjamins.

_ 2001b, Markedness and the theory of linguistic change, in H. Andersen (ed.), Actualization. Linguistic change in progress, Amsterdam-Philadelphia, Benjamins: 21-57.

Antinucci, F., Miller, R. 1976, How children talk about what happened, «Journal of child language» 3(2): 167-189.

Belardi, W. 1978, Il luogo dell'interferenza linguistica, «Incontri Linguistici» 4: 5568.

1985, Considerazioni sulla ricostruzione dell'indoeuropeo, in R. Ambrosini (ed.), Tra linguistica storica e linguistica generale. Scritti in onore di Tristano Bolelli, Pisa, Pacini Editore: 39-66.

1990, Genealogia, tipologia, ricostruzione e leggi fonetiche, in W. Belardi (ed.), Linguistica generale, filologia e critica dell'espressione, Roma, Bonacci: 155216.

_ 1993, Sulla tipologia della struttura formale della parola nelle lingue indoeuropee, «Rendiconti dell'Accademia Nazionale dei Lincei» s. 9, v. 4, f. 4: 535-570.

Berlin, B. - Kay, P. 1969, Basic color terms, Berkeley, University of California Press.

Brunel, J. 1939, L'aspect verbal et l'emploi des préverbes en grec, particulièrement en attique, Paris, C. Klincksieck.

Bybee, J.L. 1985, Morphology. A study of the relation between meaning and form, Amsterdam-Philadelphia, Benjamins.

Bybee, J., Slobin, D.I. 1982, Rules and schemas in the development and use of the English past tense, «Language» 58(2): 265-289.

Carstairs, A. 1987, Allomorphy in inflexion, London, Croom Helm.

Cennamo, M. 2001, $L$ 'extended accusative'e le nozioni di voce e relazione grammaticale nel latino tardo e medievale, in V. Viparelli (ed.), Ricerche linguistiche fra antico e moderno, Napoli, Liguori: 3-27.

Comrie, B. 1976, Aspect. Cambridge, Cambridge University Press.

Coseriu, E., 1952, Sistema, norma y habla, Montevideo, Universidad de la Republica. 1958, Sincronía, diacronía y historia, Montevideo, Universidad de la Republica. 1966, Structure lexicale et enseignement du vocabulaire, in Actes du premier 
Colloque international de Linguistique appliquée, Nancy, Faculté des Lettres e des Sciences humaines de l'Université Nancy: 175-217.

1994, Il primato della storia, in P. Cipriano - P. Di Giovine - M. Mancini (edd.), Miscellanea di studi linguistici in onore di Walter Belardi, Roma, Il Calamo: 933-955.

Croft 2000, Explaining language change: an evolutionary approach, London, Longman.

Cuzzolin, P. 1998, Tra ricostruzione e tipologia: il caso dell'ergatività indoeuropea, in G. Bernini - P. Cuzzolin - P. Molinelli (edd.), Ars linguistica. Studi offerti a Paolo Ramat, Roma, Bulzoni: 181-204.

Dardano, P. 2013, L'allineamento sintattico delle lingue indoeuropee dell'Anatolia: vecchi problemi e nuove proposte alla luce di una recente pubblicazione, «Orientalia. Nova series» 82 (2): 29-67.

Di Giovine, P. (1990-96), Studio sul Perfetto I, II, III: La posizione del perfetto all'interno del sistema verbale indoeuropeo, Roma, Il Calamo.

Dixon, R. M. W. 1994, Ergativity, Cambridge, Cambridge University Press.

Dovetto, F. 2020, Scuola tedesca, scuola francese, scuola italiana alle origini della Sprachwissenschaft, «Atti del Sodalizio Glottologico Milanese» 14: 55-84.

Drinka, B. 1999, Alignment in early Proto-Indo-European, in C.F. Justus - E.C. Polomé (eds.), Language change and typological variation. In honor of Winfred P. Lehmann on the occasion of his 83rd birthday, vol. II, Washington, Institute for the study of Man: 464-500.

Giacalone Ramat, A. 1983, Che cosa può offrire lo studio delle lingue in via di riduzione alle ricerche sull'acquisizione delle lingue straniere?, in M. Dardano - W.U. Dressler - G. Held (eds.), Parallela. Atti del $2^{\circ}$ Convegno italo-austriaco (Roma, 1-4.2.1982), Tübingen, Narr: 337-351.

2003, Verso l'italiano. Percorsi e strategie di acquisizione, Roma, Carocci.

Giannelli, L. 1973, K, P e T intervocaliche in Toscana, "Atti e Memorie dell'Accademia Toscana di Scienze e Lettere 'La Colombaria'» 38: 335-347.

Greenberg, J. 1963, Some universals of grammar with particular reference to the order of meaningful elements, in J. Greenberg (ed.), Universals of language, Cambridge MA, The MIT Press: 73-113.

Hawkins, J.A. 1979, Implicational universals as predictors of word order change, «Language» 55: 618- 648.

1983, Word order universals, New York The Academic Press.

Hoffmann, K. 1967, Der Injunktiv im Veda, Heidelberg, Winter.

Hopper, P. J., Thompson, S. A. 1980, Transitivity in grammar and discourse, «Language» 56: 251-299.

István, F. 1959, The origin of grammatical gender, «Lingua» 8: 186-214.

Jamison, S.W. 1983, Function and Form in the -áya-formations of the Rig Veda and Atharva Veda, Göttingen, Vandenhoeck \& Ruprecht.

Jasanoff, J. 1978, Stative and middle in Indo-European, (Innsbrucker Beiträge zur Sprachwissenschaft 23), Innsbruck, Institut fur Sprachwissenschaft der Universität Innsbruck. 
2003, Hittite and the Indo-European verb, Oxford, Oxford University Press.

Kiparsky, P. 1968, Linguistic universals and linguistic change, in E. Bach - R. Harms (eds.), Universals in linguistic theory, Holt, Rinehart, and Winston: 170-202.

Kulikov, L. 2012a, The Vedic -ya- presents. Passives and intransitivity in old IndoAryan, (Leiden Studies in Indo-European 19), Amsterdam-New York: Rodopi. $2012 \mathrm{~b}$, Voice and valency derivations in old Indo-Aryan in a diachronic typological perspective: the degrammaticalization of the middle and other trends in the vedic verbal system, in L. Melazzo (ed.), Usare il presente per spiegare il passato, Roma, Il Calamo: 161-191.

Kümmel, M. 1996, Stativ und Passiv aorist im Indoiranischen, Göttingen, Vandenhoeck \& Ruprecht.

Kuryłowicz, J. 1932, Les désinences moyennes de l'Indo-européen et du Hittite, «Bulletin de la Société de Linguistique de Paris» 33: 1-4.

1964, The inflectional categories of Indo-European, Heidelberg, C. Winter.

Kurzová, H. 1993, From Indo-European to Latin. The evolution of a morphosyntactic type, Amsterdam-Philadelphia, Benjamins.

— 1999, Typology and diachrony of the middle voice, in S. Embleton et alii (eds.), The Emergence of the Modern Language Sciences: Studies on the transition from historical-comparative to structural linguistics in honour of E.F.K. Koerner, Amsterdam, Benjamins: 115-129.

2011, Notes on Indo-European participles, «Archivio Glottologico Italiano» 96 (2): 146-178.

2014, Diathesis and transitivity in Latin and Greek: chronology of Indo-European voice types, «Listy filologické» 137 (3-4): 217-243.

Labov, W. 2001, Principles of linguistic change: Social factors, (Language in Society 29), Malden, MA, Blackwell Publishers.

Lanman, C. R. 1880, A statistical account of noun-inflection in the Veda, «Journal of American Oriental Society» 10: 325-602.

Lazzeroni, R. 1956, Considerazioni sull'aspetto verbale in frase negativa nel greco classico, «Annali della Scuola Normale Superiore di Pisa» 25 (3-4): 213-233.

1957, L'aspetto verbale con gli avverbi di rapidità e con quelli significanti «improvvisamente» nel greco classico, «Annali della Scuola Normale Superiore di Pisa», 26 (1-2): 88-97.

— 1960, Considerazioni sulla cronologia di alcune isoglosse delle lingue anatoliche, "Annali della Scuola Normale Superiore di Pisa», 29 (1-2): 103124.

— 1967, Su alcune correnti dialettali nel Peloponneso antico, «Studi e Saggi Linguistici» 7: 63-75.

1968, Per una definizione dell'unità indoiranica, «Studi e Saggi Linguistici»8: 131-159.

1969, Stratificazioni nella lingua poetica greca, in G. Bolognesi et al. (edd.), Studi linguistici in onore di Vittore Pisani, vol. 2, Brescia, Paideia: 619-634. 1972, Contatti di lingue e culture nell'Italia antica: elementi greci nei dialetti italici, «Studi e Saggi Linguistici»12: 1-24. 
1977, Fra glottogonia e storia: ingiuntivo, aumento e lingua poetica indoeuropea, «Studi e Saggi Linguistici» 17: 1-30.

1978, Fra glottogonia e storia: $i$ verbi sanscriti della VI classe, «Studi e Saggi Linguistici» 18: 129-148.

1979, Fra glottogonia e storia: ingiuntivo e indicativo in una desinenza verbale sanscrita, «Studi e Saggi Linguistici» 19: 53-66.

1980, Fra glottogonia e storia: ipotesi sulla formazione del sistema verbale sanscrito, «Studi e Saggi Linguistici» 20: 23-53.

1982, Frase nominale e ingiuntivo nel Rig Veda, «Studi Classici e Orientali» 32: 227-283.

1983a, Su una peculiarità della flessione atematica greca, «Studi e Saggi Linguistici» 23: 15-21.

1983b, Contatti di lingue e culture nell'Italia antica. Modelli egemoni e modelli subordinati nelle iscrizioni osche in grafia greca, "Annali dell'Istituto Universitario Orientale di Napoli - Sezione Linguistica» 5: 171-182.

_ 1984a, La formazione del sistema dei tempi e degli aspetti nel verbo sanscrito, «Atti del Sodalizio Glottologico Milanese» 24: 55-63.

_ 1984b, Lingua e società in Atene antica. La crisi linguistica del V secolo, «Studi Classici e Orientali» 34: 13-25.

— 1985a, La flessione tematica greca e le relazioni di persona nel verbo indoeuropeo, in R. Ambrosini (ed.), Tra linguistica storica e linguistica generale. Scritti in onore di Tristano Bolelli, Pisa, Pacini editore: 165-179.

1985b, Sistema verbale sanscrito e sistema verbale indoeuropeo: le desinenze del congiuntivo vedico, in E. Campanile - R. Lazzeroni - R. Peroni (edd.), Scritti in onore di Riccardo Ambrosini, Pisa, Giardini Editore: 129-133.

_ 1987a, Gli ottativi vedici del tipo gaméma e le forme modali autonome indoeuropee, «Studi e Saggi Linguistici» 27: 123-150.

1987b, Il mutamento linguistico, in R. Lazzeroni (ed.), Linguistica storica, Roma, La Nuova Italia: 13-54.

1989, Congiuntivo indoeuropeo e indicativo germanico. Ipotesi sulla formazione della flessione tematica indoeuropea, «Romano-Barbarica» 9: 199-215.

1990, La diatesi come categoria linguistica: studio sul medio indoeuropeo, «Studi e Saggi Linguistici» 30: 1-22.

1991a, Contatti di lingue e di culture nell'Italia antica. Un bilancio, in E. Campanile (ed.), Rapporti linguistici e culturali tra i popoli dell'Italia antica (Pisa, 6-7 ottobre 1989), Pisa, Giardini: 177-188.

1991b, Mutamento morfologico e diffusione lessicale. Il contributo del sanscrito, in M. Negri - F. Aspesi (edd.), Studia linguistica amico et magistro oblata. Scritti di amici e allievi dedicati alla memoria di Enzo Evangelisti, Milano, Unicopli. 205-217.

_ 1992a, Genere grammaticale e sistema nominale nel sanscrito. Per una interpretazione della deriva, «Archivio Glottologico Italiano» LVII: 89-104. 1992b, L'espressione dell'agente come categoria linguistica. I nomi indoeuropei in-tér/-tor, «Studi e Saggi Linguistici» 32: 233-245. 
1993a, Il genere indoeuropeo. Una categoria naturale?, in M. Bettini (ed.), Maschile/Femminile. Generi e ruoli nelle culture antiche, Bari, Laterza: 3-16.

-1993b, Arcaismi e innovazioni nel sistema verbale vedico. Le forme dello stativo, «Studi e Saggi Linguistici» 33: 11-23.

1994, Rileggendo Benveniste: le relazioni di persona nel verbo, in S. Giannini R. Lazzeroni (edd.), Linguistic Categorization, «Rivista di Linguistica» 6 (2): 267-274.

1995a, La baritonesi come segno dell'individuazione: il caso del vocativo indoeuropeo, «Studi e Saggi Linguistici» 35: 33-44.

1995b, Categorizzazioni linguistiche, in R. Ajello - S. Sani (edd.), Studi linguistici e filologici in onore di Tristano Bolelli, Pisa, Pacini Editore: 283-292.

_ 1996, Statività e modalità. Il caso del sanscrito, «Studi e Saggi Linguistici» 36: 127-139.

1997, La transitività come categoria linguistica: i nomi d'azione indoeuropei, «Incontri Linguistici» 20: 71-82.

1998, La cultura indoeuropea, Bari, Laterza.

1999a, I dialetti greci fra isoglosse e varianti, in P. Berrettoni (ed.), Varietà linguistiche nella storia della grecità. Atti del terzo incontro internazionale di linguistica greca (Pisa, 2-4 Ottobre 1997), Alessandria, Edizioni dell'Orso: 139144.

1999b, A cultura indo-europeia, Lisboa, Editorial Estampa (trad. portoghese di I.T. Santos).

2000a, Congiuntivo e indicativo. Una vicenda sanscrita e (forse) indoeuropea, «Studi e Saggi Linguistici» 38: 89-100.

2000b, Genere grammaticale e riorganizzazione dei paradigmi: il caso della IV declinazione latina, «Archivio Glottologico Italiano» 85 (2): 232-237.

2002a, Transitivi, causativi e incoativi nel sistema verbale vedico, «Incontri Linguistici» 25: 105-122.

2002b, Il nome greco del sogno e il neutro indoeuropeo, «Archivio Glottologico Italiano», 87 (2): 145-162.

_-2002c, Ruoli tematici e genere grammaticale: un aspetto della morfosintassi indoeuropea?, «Archivio Glottologico Italiano» 87 (1): 3-19.

2004, Inaccusatività indoeuropea e alternanza causativa vedica, «Archivio Glottologico Italiano», 89 (2): 139-164.

2005, Mutamento e apprendimento, in L. Costamagna - S. Giannini (edd.), Acquisizione e mutamento di categorie linguistiche. Atti del convegno della Società Italiana di Glottologia (Perugia, 23-25 ottobre 2003), Roma, Il Calamo: 13-24.

2006, Il dialetto di Sparta fra cedimento e restaurazione, «Incontri Linguistici» 29: 83-89.

2007, Il vedico fra varianti e standardizzazione, in P. Molinelli (ed.), Standard e non standard tra scelta e norma. Atti del XXX convegno della Società Italiana di Glottologia (Bergamo, 20-22 ottobre 2005), Roma, Il Calamo: 109-116.

_ 2008, Alternanza causativa e classi di presente in vedico, contributo alla 
soluzione dell'"enigma" di Kuiper, in L. Kulikov - M. Rusanov (eds.), Indologica. T. Ya. Elizarenkova Memorial Volume. Vol. 1, Moscow, Russian State University for the Humanities: 263-272.

2009, Causativi e transitivi indoeuropei: fra comparazione e tipologia, «Studi e Saggi Linguistici» 47: 7-23.

2011a, Walter Belardi indoeuropeista, in Atti del Convegno in ricordo di Walter Belardi (Roma, 12 novembre 2009), Roma, Accademia Nazionale dei Lincei: 57-65.

_ 2011b, Classi di presente e raddoppiamento in alcune lingue indoeuropee, «Archivio Glottologico Italiano» 96 (2): 129-145.

$2011 \mathrm{c}$, Macrocategorie o trasformazione di categorie? Dal perfetto indoeuropeo alla coniugazione in -hi dell'ittita, «Incontri Linguistici» 34: 47-70.

2012a, Scala o scale di nominalità? Il caso dei nomi d'azione vedici, «Archivio Glottologico Italiano» 97 (2): 145-159.

2012b, L'espansione del latino nell'Italia antica. Contatti e conflitti di lingue e di culture, in R. Bombi - V. Orioles (ed.), 150 Anni. L'identità linguistica italiana. Atti del XXXVI convegno della Società Italiana di Glottologia (Udine, 27-29 Ottobre 2011), Roma, Il Calamo, Roma: 15-27.

2013a, Fra ruoli semantici e ruoli pragmatici: il cosiddetto «schema pindarico» nel greco antico, «Archivio Glottologico Italiano» 98 (1): 26-40.

2013b, I percorsi del mutamento: categorie scalari e sincretismo degli ausiliari, «Studi e Saggi Linguistici» 51 (1): 33-52.

2014, L'attuazione di un mutamento: perfetto e medio in alcune lingue indoeuropee, «Archivio Glottologico Italiano» 99 (2): 129-154.

2015a, Un paradosso di Wackernagel: diatesi media e desinenze personali attive. Una possibile soluzione, «Archivio Glottologico Italiano» 100 (1): 3-18.

2015b, Divagazioni sulla legge degli appellativi in greco e in vedico, in G.

Marotta - F. Rovai (edd.), Ancient languages between variation and norm, «Studi e Saggi Linguistici» 53 (2): 419-428.

2015 c, Fra mutamento linguistico e organizzazione della memoria: la partizione del paradigma in alcune lingue indoeuropee, in M.G. Busà - S. Gesuato (edd.), Lingue e contesti. Studi in onore di Alberto M. Mioni, Padova, CLEUP: 125142.

_ 2016a, Statività e modalità: i modi del perfetto, «Studi e Saggi Linguistici» 54 (1): 7-25.

2016b, Geolinguistica, sociolinguistica e cronologia: le dimensioni del mutamento, «Atti del Sodalizio Glottologico Milanese» 9: 269-276.

2017a, I presenti in -ya nel Rig Veda: alla ricerca dell'intransitività scissa, «Archivio Glottologico Italiano» 102 (1): 3-19.

_ 2017b, Divagazioni sull'aumento in Omero, in G. Marotta - F. Strik Lievers (edd.), Strutture linguistiche e dati empirici in diacronia e sincronia, Pisa, Pisa University Press: 33-56.

2017 c, I causativi vedici fra semantica e morfologia, in R. D'Alessandro - G. Iannàcaro - D. Passino - A. Thornton (edd.), Di tutti i colori. Studi linguistici 
per Maria Grossmann, Utrecht, Utrecht University Repository: 185-195.

2017d, Il nominativo esclamativo latino: un Eï $\delta \omega \lambda$ ov scholae?, «Incontri Linguistici» 40: 77-89.

2018a, Il mutamento linguistico dall'800 ai nostri giorni, in F. Da Milano - A.

Scala - M. Vai - R. Zama (edd.), La cultura linguistica italiana in confronto con le culture linguistiche di altri paesi europei dall'Ottocento in poi. Atti del L Congresso internazionale di studi della Società di Linguistica Italiana (Milano, 22-24 settembre 2016), Roma, Bulzoni: 13-29.

2018b, Jakobson e la nozione di marcatezza. Riflessioni di un indoeuropeista, in

E. Esposito - S. Sini - M. Castagneto (edd.), Roman Jakobson. Linguistica e poetica, Milano, Ledizioni: 316-324.

2019a, Ricordo di Aldo Luigi Prosdocimi, «Studi e Saggi Linguistici» 57 (1): 714.

2019b, Divagazioni conviviali su deriva, marcatezza e memoria, in M. Pastore Stocchi (ed.), I deipnosofisti lincei. Omaggio a Maurizio Vitale, Roma-Padova, Antenore: 175-194.

_2019c, Descrizione e spiegazione nella linguistica storica, «Atti del Sodalizio Glottologico Milanese» 13: 145-152.

2020, Il mutamento linguistico fra contatto, irradiazione e memoria, in P. Del Puente - F. Guazzelli - L. Molinu - S. Pisano, Tra etimologia romanza e dialettologia. Studi in onore di Franco Fanciullo, Alessandria, Edizioni dell'Orso: 191-202.

Lindstedt, J. 1995, Understanding perfectivity - understanding bounds, in P. M. Bertinetto - V. Bianchi - Ö. Dahl - M. Squartini (eds.), Temporal reference, aspect and actionality: Typological perspectives. Vol. 2, Torino, Rosenberg \& Sellier: 239 - 247.

Maffei, L. 2018, Elogio della parola, Bologna, Il Mulino.

Mancini, M. 2008, Appunti sulla circolazione del latino nella Palestina del I secolo d.C., in R. Lazzeroni - E. Banfi - G. Bernini - M. Chini - G. Marotta (edd.), Diachronica et Synchronica. Studi in onore di Anna Giacalone Ramat, Pisa, ETS: 277-299.

_-2013, L'epigrafia giudaica e la diffusione del greco nella Palestina romana, in M. Mancini - L. Lorenzetti (edd.), Le lingue del Mediterraneo antico. Culture, mutamenti, contatti, Roma, Carocci: 213-259.

_2020, La parte più durevole del nostro passato: un ricordo di Romano Lazzeroni, «Studi e Saggi Linguistici», supplemento al volume 58 (2): 19-27.

Manzini, M. R. - Savoia, L.M. 2011, Grammatical Categories. Variation in romance languages, Cambridge, Cambridge University Press.

Martinet, A. 1955, Économie des changements phonétiques, Berne, Éditions A. Francke S.A.

Milizia, P. 2013, L'equilibrio nella codifica morfologica, Roma, Carocci.

Narten, J. 1964, Die sigmatischen Aoriste im Veda, Wiesbaden, Otto Harrassowitz.

— 1968, Zum «Proterodynamischen» Wurzelpräsens, in J.C. Heesterman - G.H. Schokker - V.I. Subramoniam (eds.), Pratidanam: Indian, Iranian, and Indo- 
European studies presented to Franciscus Bernardus Jacobus Kuiper on his sixtieth birthday, Berlin-Boston, De Gruyter Mouton: 9-19.

Neu, E. 1968, Das hethitische Mediopassiv und seine indogermanischen Grundlagen, Wiesbaden, Harrassowitz.

Oettinger, N. 1976, Der Indogermanische Stativ, «Münchener Studien zur Sprachwissenschaft» 34: 109-149.

Paul, H. 1880, Prinzipien der Sprachgeschichte. Band I, Halle a.S., Max Niemeyer.

Pooth, R., Kerkhof, P., Kulikov, L., Bardall, J. 2019, The origin of non-canonical case marking of subjects in Proto-Indo-European. Accusative, ergative or semantic alignment, (Indogermanische Forschungen 124,1), Berlin-New York, De Gruyter: 245-264.

Ramat, P. 1985, On the scalar character of (morphological) irregularity, in H. Seiler - G. Brettschneider (eds.), Language Invariants and Mental Operations. Internat. Interdisc. Confer. held at Gummersbach/Cologne, Germany, sept. 1823 1983, Narr, Tübingen: 162-71.

Rohlfs, G. 1949-1954, Historische Grammatik der italienischen Sprache und ihrer Mundarten. Band I-II-III, Bern, A. Franke Verlag.

Romagno, D. 2002, Diatesi indoeuropea e verbi di movimento greci: alcune considerazioni sull'intransitività, «Archivio Glottologico Italiano» 87 (2): 163174.

2005, Il perfetto omerico. Diatesi, azionalità e ruoli tematici, Milano, Franco Angeli.

2011, Codifica argomentale e ruoli semantici. Ergativo/accusativo vs. attivo, «Archivio Glottologico Italiano» 96 (1): 3-30.

2014, The aorist in - $\eta v$ in Homeric Greek: at the morphosyntax/semantics interface. A thorough analysis of Iliad and Odyssey, "Archivio Glottologico Italiano» 99 (2): 155-186.

2015, Telicità inerente e telicità configurazionale: l'uso del clitico dativo in alcune varietà dialettali della Calabria settentrionale, «L'Italia Dialettale» 76: 163-179.

_ 2021, The extension of a linguistic category: middle voice in homeric Greek between subject affectedness, reflexivity and possession, «Archivio Glottologico Italiano» 106 (1): 3-42.

Rosch, E. 1973, Natural categories, «Cognitive Psychology» 4: 328-350.

— 1978, Principles of categorization, in E. Rosch - B.B. Lloyd (eds.), Cognition and Categorization, Hillsdale, Lawrence Erlbaum: 27-48.

Rosch, E. - Mervis, C.B. 1975, Family resemblances. Studies in the internal structure of categories, «Cognitive Psychology» 7 (4): 573-605.

Rose, S. 2006, The Hittite -hi/-mi Conjugations, Innsbruck, Institute für Sprache und Literatur der Universität.

Rumsey, A. 1987, The chimera of Proto-Indo-European ergativity, «Lingua» 71: 297 318.

Sapir, E. 1921, Language. An introduction to the study of speech, New York Harcourt, Brace \& World Inc. 
Sasse, H. J. 1992, Language decay and contact-induced change. Similarities and differences, in M. Brenzinger (ed.), Language death, Berlin/New York, Mouton de Gruyter: 59-80.

Slabakova, R. 2001, Telicity in the second language, Amsterdam-Philadelphia, Benjamins.

Song, C. 2018, Severing telicity from result. On two types of resultative compound verb in Dongying Mandarin, «Journal of East Asian Linguistics» 27: 275-307.

Silverstein, M. 1976, Hierarchy of features and ergativity, in R.M.W. Dixon (ed.), Grammatical categories in australian languages, Canberra, Australian Institute of Aboriginal Studies: 112-171.

Stang, C. 1932, Perfektum und Medium, «Norsk tidsskrift for sprogvidenskap» 6: 2939.

Taylor, J. R. 1989, Linguistic categorization: prototypes in linguistic theory, Oxford, Clarendon Press.

Thurneysen, R. 1885, Der indogermanische imperativ, «Zeitschrift für vergleichende Sprachforschung auf dem Gebiete der Indogermanischen Sprachen» 27 (2): 172180.

Timberlake, A. 1977, Reanalysis and actualization of syntactic change, in C. N. Li (ed.), Mechanisms of syntactic change, Austin-London, University of Texas Press: 141-177.

Trudgill, P. 1983, On dialect. Social and geographical perspectives, Oxford, Blackwell. Uhlenbeck, C. 1901, Agens und Patiens im Kasussystem der indogermanischen Sprachen, «Indogermanische Forschungen» 12: 170-172.

Vendler, Z. 1967, Linguistics in Philosophy. Ithaca (NY): Cornell University Press.

Vendryes, J. 1946, La comparaison en linguistique, "Bulletin de la société de linguistique de Paris» 124 (1): 1-16.

Villar, F, 1984, Ergativity and animate/inanimate gender in Indo-European, «Zeitschrift für vergleichende Sprachforschung» 97: 167-196.

Wackernagel, J. 1904, Studien zum griechischen Perfektum. Kleine Schriften II, Göttingen, Vandenhoeck \& Ruprecht.

— 1926, Vorlesungen über Syntax, Basel, Birkhäuser.

Watkins, C. 1969, Indogermanische Grammatik III/1, Heidelberg, Winter.

Weinreich, U. - Labov, W. - Herzog, M.I. 1968, Empirical foundations for a theory of language change, in W.P. Lehmann - Y. Malkiel (eds.), Directions for Historical Lingusitics, Austin, University of Texas Press: 95-195.

Willis, D. 2013, Negation in the history of the Slavonic languages, in D. Willis (ed.), The history of negation in the languages of Europe and the Mediterranean. Vol. 1: Case studies, Oxford, Oxford University Press: 341-398.

Wittgenstein, L. 1953, Philosophische Untersuchungen, Frankfurt am Main, Suhrkamp.

Wurzel, W. 1984, Flexionsmorphologie und Natuerlichkeit, Berlin, Akademie-Verlag. 1989, Inflectional morphology and naturalness, Dordrecht, Kluwer. 